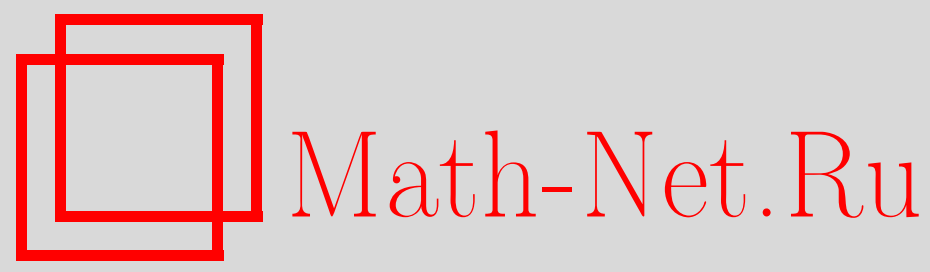

М. В. Иванова, В. И. Ушаков, Вторая краевая задача для псевдопараболического уравнения в нецилиндрической области, Матем. заметки, 2002, том 72, выпуск 1, 48-53

DOI: https://doi.org/10.4213/mzm403

Использование Общероссийского математического портала Math-Net.Ru подразумевает, что вы прочитали и согласны с пользовательским соглашением http://www.mathnet.ru/rus/agreement

Параметры загрузки:

IP: 18.234 .156 .22

26 апреля 2023 г., 03:01:18

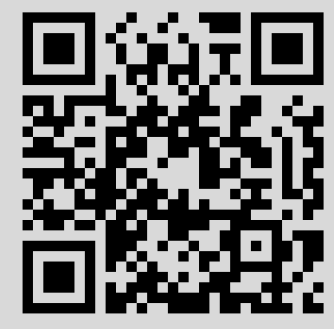




\title{
ВТОРАЯ КРАЕВАЯ ЗАДАЧА ДЛЯ ПСЕВДОПАРАБОЛИЧЕСКОГО УРАВНЕНИЯ В НЕЦИЛИНДРИЧЕСКОЙ ОБЛАСТИ
}

\author{
М.В. Иванова, В. И. Ушаков
}

Работа посвящена исследованию разрешимости второй смешанной задачи в нецилиндрической области для нестационарного уравнения

$$
\operatorname{div}\left(k(x) \operatorname{grad} u_{t}\right)-c(x) u_{t}-b(x) u(x, t)=f(x, t),
$$

называемого псевдопараболическим уравнением. Доказываются теоремы существования и единственности решения в случае сужаюшейся при возрастании времени $t$ области.

Библиограффия: 4 названия.

В настоящей статье изучаются вопросы существования и единственности решения псевдопараболического уравнения

$$
\operatorname{div}\left(k(x) \operatorname{grad} u_{t}\right)-c(x) u_{t}=b(x) u(x, t)+f(x, t),
$$

в нецилиндрической области.

Такого вида уравнения возникают в теории фильтрации жидкости (уравнения Баренблатта-Желтова-Кочиной), в теории влагопереноса в почве (уравнение Аллера), теории теплопроводности в среде с “двумя температурами” (уравнение Чена-Гуртина) и многих других.

Изучению краевых задач для псевдопараболических уравнений в цилиндрических областях посвящено большое число работ (например, [1], [2]). Краевые задачи в нецилиндрических областях для параболического уравнения были рассмотрены одним из авторов в работе [3]. В данной работе рассматривается случай, когда задача поставлена для данного уравнения в “сужающейся" области; также был исследован случай "расширяющейся" области [4].

Рассмотрим область $Q \subset \mathbb{R}^{n+1}$. Обозначим через $\Omega_{\tau}$ проекцию сечения области $Q$ плоскостью $t=\tau, Q_{T}=\{(x, t) \in Q \mid t \in[0, T]\}$ на координатную плоскость $\{t=0\}$.

Напомним стандартные определения: $W_{2}^{1,0}\left(Q_{T}\right)$ - гильбертово пространство, состоящее из элементов $f(x, t)$ пространства $L_{2}\left(Q_{T}\right)$, имеющих квадратично суммируемые по $Q_{T}$ обобщенные производные $\partial f / \partial x_{i}, i=1, \ldots, n$.

Норма в этом пространстве

$$
\|f(x, t)\|_{W_{2}^{1,0}\left(Q_{T}\right)}^{2}=\int_{Q_{T}}\left(f^{2}(x, t)+(\nabla f(x, t))^{2}\right) d x d t
$$


где $W_{2}^{0,1}\left(Q_{T}\right)$ - гильбертово пространство, состоящее из элементов $f(x, t)$ пространства $L_{2}\left(Q_{T}\right)$, имеющих квадратично суммируемые по $Q_{T}$ обобщенные производные $\partial f / \partial t$ с нормой

$$
\|f(x, t)\|_{W_{2}^{0,1}\left(Q_{T}\right)}^{2}=\int_{Q_{T}}\left(f^{2}(x, t)+\left(\frac{\partial f}{\partial t}\right)^{2}\right) d x d t .
$$

Будем говорить, что область $Q_{T}$ сужается, если при любых $t_{1}, t_{2}$ таких, что $0 \leqslant t_{1} \leqslant$ $t_{2} \leqslant T, \Omega_{t_{2}} \subset \Omega_{t_{1}}\left(\Omega_{0}\right.$ предполагается не пустой и ограниченной).

Рассмотрим краевую задачу в “криволинейном цилиндре” $Q_{T}$ для псевдопараболического уравнения с переменньми коэффициентами:

$$
\begin{gathered}
\operatorname{div}\left(k(x) \operatorname{grad} u_{t}(x, t)\right)-c(x) u_{t}(x, t)=b(x) u(x, t)+f(x, t), \\
u(x, 0)=\varphi(x),\left.\quad \frac{\partial u_{t}}{\partial n}\right|_{\Gamma}=0,
\end{gathered}
$$

где $\Gamma$ - боковая поверхность $Q_{T}, \Gamma=(x, t) \in \partial Q \mid t \in(0, T)$ a $n$ - внешняя нормаль к $\Omega_{t}$.

Потребуем вьполнения дополнительных условий:

(i) функция $\varphi(x) \in L_{2}\left(\Omega_{0}\right)$ такая, что $\nabla \varphi(x) \in L_{2}\left(\Omega_{0}\right)$, функция $f(x, t) \in L_{2}\left(Q_{T}\right)$;

(ii) функции $b(x), c(x), k(x)$ измеримые на $\Omega_{0}$ и такие, что $\forall x \in \Omega_{0}$

$$
0<b \leqslant b(x) \leqslant B, \quad 0<c \leqslant c(x) \leqslant C, \quad 0<k \leqslant k(x) \leqslant K
$$

(iii) часть границы $Q_{T}$, лежащую в полупространстве $t>0$, можно представить в виде $\Gamma=\left\{(x, \psi(x)) \mid x \in \Omega_{0}\right\}, \psi(x): \mathbb{R}^{n} \rightarrow \mathbb{R}$, где $\psi(x)$ - гладкая функция, $\nabla \psi(x) \neq 0$.

Целью работы является доказательство существования и единственности обобщенного решения этой задачи.

Обобщенным решением задачи $(1)-(3)$ в $Q_{T}$ будем считать функцию $u(x, t) \in$ $W_{2}^{0,1}\left(Q_{T}\right)$, для которой $u_{t}(x, t) \in W_{2}^{1,0}\left(Q_{T}\right)$, удовлетворяющую интегральному тождеству

$$
\begin{gathered}
-\int_{Q_{T}} k(x) \nabla u_{t}(x, t) \nabla v(x, t) d x d t-\int_{Q_{T}} c(x) v(x, t) u_{t}(x, t) d x d t \\
=\int_{Q_{T}} b(x) v(x, t) u(x, t) d x d t+\int_{Q_{T}} f(x, t) v(x, t) d x d t
\end{gathered}
$$

для любой пробной функции $v(x, t) \in W_{2}^{1,0}\left(Q_{T}\right)$ и удовлетворяющую начальному условию (2).

ТеоремА. Пусть область $Q_{T}$ сужается и выполняются условия (i), (ii), (iii). Тогда решение задачи (1)-(3) существует и единственно. 
ДокАЗАТЕЛЬСТВо. Пусть $\left\{\varphi_{k}(x)\right\}_{k=1}^{\infty}-$ ортонормированный базис в $L_{2}\left(\Omega_{0}\right)$ такой, что для всех $t \in[0, T]$ система $\varphi(x)$ линейно независима в $L_{2}\left(\Omega_{t}\right)$. Например, систему $\varphi(k)$ можно получить процессом ортогонализации из системы одночленов $\left\{x_{1}^{\alpha_{1}}, x_{2}^{\alpha_{2}}\right.$, $\left.x_{3}^{\alpha_{3}}, \ldots, x_{n}^{\alpha_{n}}\right\}$, где $\alpha_{i}$ целые неотрицательные числа. Отметим, что $\forall \tau \in[0, T]$ система $\left\{\varphi_{k} \mid \Omega_{\tau}\right\}_{k=1}^{\infty}$ является базисом в $L_{2}\left(\Omega_{\tau}\right)$ (не обязательно, конечно, ортонормированным).

Приближенное решение будем искать в виде

$$
u^{N}(x, t)=\sum_{k=1}^{N} c_{k}^{N}(t) \varphi_{k}(x)
$$

где коэффициенты $c_{k}^{N}(t)$ удовлетворяют системе дифференциальных уравнений

$$
\begin{aligned}
& -\sum_{k=1}^{N} \dot{c}_{k}^{N}(t) \int_{\Omega_{t}}\left(k(x) \nabla \varphi_{k}(x) \nabla \varphi_{i}(x)+c(x) \varphi_{k}(x) \varphi_{i}(x)\right) d x \\
& \quad=\sum_{k=1}^{N} c_{k}^{N}(t) \int_{\Omega_{t}} b(x) \varphi_{k}(x) \varphi_{i}(x) d x+\int_{\Omega_{t}} f(x, t) \varphi_{i}(x) d x, \quad i=1, \ldots, N,
\end{aligned}
$$

с начальньми условиями

$$
c_{i}(0)=\left(\varphi(x), \varphi_{i}(x)\right)_{L_{2}\left(\Omega_{0}\right)}, \quad i=1, \ldots, N
$$

Получили линейную неоднородную систему из $N$ дифференциальных уравнений с переменными коэффициентами относительно $N$ неизвестных $c_{i}(t)$ :

$$
\begin{aligned}
\sum_{k=1}^{N} \alpha_{k i}(t) \dot{c}_{k}(t) & =\sum_{k=1}^{N} \beta_{k i}(t) c_{k}(t)+f_{i}(t), \quad i=1, \ldots, N \\
\alpha_{k i}(t) & =-\int_{\Omega_{t}}\left(k(x) \nabla \varphi_{k}(x) \nabla \varphi_{i}(x)+c(x) \varphi_{k}(x) \varphi_{i}(x)\right) d x \\
\beta_{k i}(t) & =\int_{\Omega_{t}} b(x) \varphi_{k}(x) \varphi_{i}(x) d x, \quad f_{i}(t)=\int_{\Omega_{t}} f(x, t) \varphi_{i}(x) d x
\end{aligned}
$$

причем матрица $\left\{a_{i j}\right\}$ обратима в силу выбора системы функций $\left\{\varphi_{k}\right\}$. Следовательно, система дифференциальных уравнений (7) с начальными условиями (6) имеет единственное решение. Это решение определено на всем отрезке $[0, T]$.

Покажем ограниченность норм $\left\|u^{N}(x, t)\right\|_{W_{2}^{0,1}\left(Q_{T}\right)},\left\|u_{t}^{N}(x, t)\right\|_{W_{2}^{1,0}\left(Q_{T}\right)}$. Для этого умножим уравнение $(5)$ на $c_{i}(t)$, просуммируем от 1 до $N$ и проинтегрируем от 0 до $T$ :

$$
\begin{gathered}
-\int_{Q_{T}}\left(k(x) \nabla u_{t}^{N}(x, t) \nabla u^{N}(x, t)+c(x) u_{t}^{N}(x, t) u^{N}(x, t)\right) d x d t \\
=\int_{Q_{T}} b(x)\left(u^{N}(x, t)\right)^{2} d x d t+\int_{Q_{T}} f(x, t) u^{N}(x, t) d x d t
\end{gathered}
$$


Учитьвая (ii) и интегрируя неравенства по времени, получим

$$
\begin{aligned}
\left\|u^{N}(x, t)\right\|_{L_{2}\left(Q_{T}\right)}^{2} \leqslant & \frac{K}{b}\|\nabla \varphi(x)\|_{L_{2}\left(\Omega_{0}\right)}^{2}+\frac{C}{b}\|\varphi(x)\|_{L_{2}\left(\Omega_{0}\right)}^{2}+\frac{1}{b^{2}}\|f(x, t)\|_{L_{2}\left(Q_{T}\right)}^{2} \\
\left\|\nabla u^{N}(x, t)\right\|_{L_{2}\left(Q_{T}\right)}^{2} \leqslant & \frac{K T}{k}\left(1+\frac{1}{b}\right)\|\nabla \varphi(x)\|_{L_{2}\left(\Omega_{0}\right)}^{2}+\frac{C T}{k}\left(1+\frac{1}{b}\right)\|\varphi(x)\|_{L_{2}\left(\Omega_{0}\right)}^{2} \\
& +\frac{T}{k}\left(\frac{1}{b^{2}}+\frac{1}{8}\right)\|f(x, t)\|_{L_{2}\left(Q_{T}\right)}^{2} .
\end{aligned}
$$

Умножив уравнение $(5)$ на $\dot{c}_{i}(t)$, просуммируем от 1 до $N$ и проинтегрируем полученное выражение от 0 до $T$; получим

$$
\begin{aligned}
& -\int_{Q_{T}}\left(k(x)\left(\nabla u_{t}^{N}(x, t)\right)^{2}+c(x)\left(u_{t}^{N}(x, t)\right)^{2}\right) d x d t \\
& \quad=\int_{Q_{T}} b(x) u_{t}^{N}(x, t) u^{N}(x, t) d x d t+\int_{Q_{T}} f(x, t) u_{t}^{N}(x, t) d x d t
\end{aligned}
$$

\section{Отсюда}

$$
\begin{aligned}
\left\|u_{t}^{N}(x, t)\right\|_{L_{2}\left(Q_{T}\right)}^{2} & \leqslant \frac{B}{c}\|\varphi(x)\|_{L_{2}\left(\Omega_{0}\right)}^{2}+\frac{1}{c^{2}}\|f(x, t)\|_{L_{2}\left(Q_{T}\right)}^{2}, \\
\left\|\nabla u_{t}^{N}(x, t)\right\|_{L_{2}\left(Q_{T}\right)}^{2} & \leqslant \frac{B}{k}\left(\frac{1}{2}+\frac{1}{c}\right)\|\varphi(x)\|_{L_{2}\left(\Omega_{0}\right)}^{2}+\frac{1}{k}\left(\frac{1}{4}+\frac{1}{c^{2}}\right)\|f(x, t)\|_{L_{2}\left(Q_{T}\right)}^{2} .
\end{aligned}
$$

На основе полученных оценок можем оценить интересующие нас нормы:

$$
\begin{aligned}
& \left\|u^{N}(x, t)\right\|_{W_{2}^{0,1}\left(Q_{T}\right)}^{2}=\left\|u^{N}(x, t)\right\|_{L_{2}\left(Q_{T}\right)}^{2}+\left\|u_{t}^{N}(x, t)\right\|_{L_{2}\left(Q_{T}\right)}^{2} \\
& \quad \leqslant \frac{K}{b}\|\nabla \varphi(x)\|_{L_{2}\left(\Omega_{0}\right)}^{2}+\left(\frac{C}{b}+\frac{B}{c}\right)\|\varphi(x)\|_{L_{2}\left(\Omega_{0}\right)}^{2}+\left(\frac{1}{b^{2}}+\frac{1}{c^{2}}\right)\|f(x, t)\|_{L_{2}\left(Q_{T}\right)}^{2}, \\
& \left\|u_{t}^{N}(x, t)\right\|_{W_{2}^{1,0}\left(Q_{T}\right)}^{2}=\left\|u_{t}^{N}(x, t)\right\|_{L_{2}\left(Q_{T}\right)}^{2}+\left\|\nabla u_{t}^{N}(x, t)\right\|_{L_{2}\left(Q_{T}\right)}^{2} \\
& \quad \leqslant B\left(\frac{1}{2 k}+\frac{1}{k c}+\frac{1}{c}\right)\|\varphi(x)\|_{L_{2}\left(\Omega_{0}\right)}^{2}+\left(\frac{1}{c^{2} k}+\frac{1}{4 k}+\frac{1}{c^{2}}\right)\|f(x, t)\|_{L_{2}\left(Q_{T}\right)}^{2}
\end{aligned}
$$

и, кроме этого,

$$
\begin{aligned}
& \left\|u^{N}(x, t)\right\|_{W_{2}^{1}\left(Q_{T}\right)}^{2}=\left\|u^{N}(x, t)\right\|_{L_{2}\left(Q_{T}\right)}^{2}+\left\|u_{t}^{N}(x, t)\right\|_{L_{2}\left(Q_{T}\right)}^{2}+\left\|\nabla u^{N}(x, t)\right\|_{L_{2}\left(Q_{T}\right)}^{2} \\
& \leqslant\left(\frac{K T}{k}+\frac{K}{b}+\frac{K T}{k b}\right)\|\nabla \varphi(x)\|_{L_{2}\left(\Omega_{0}\right)}^{2}+\left(\frac{C T}{k}+\frac{C T}{b k}+\frac{C}{b}+\frac{B}{c}\right)\|\varphi(x)\|_{L_{2}\left(\Omega_{0}\right)}^{2} \\
& \quad+\left(\frac{T}{8 k}+\frac{T}{b^{2} k}+\frac{1}{b^{2}}+\frac{1}{c^{2}}\right)\|f(x, t)\|_{L_{2}\left(Q_{T}\right)}^{2},
\end{aligned}
$$

т.е. нормы $u^{N}(x, t)$ и $u_{t}^{N}(x, t)$ ограничены независимо от $N$. Следовательно, можно выбрать подпоследовательность $\left\{u^{N_{k}}(x, t)\right\}_{k=1}^{\infty}$ такую, что

$$
u^{N_{k}}(x, t) \rightarrow u(x, t) \quad \text { в } W_{2}^{0,1}\left(Q_{T}\right), \quad u_{t}^{N_{k}}(x, t) \rightarrow u_{t}(x, t) \text { в } W_{2}^{1,0}\left(Q_{T}\right)
$$


к некоторой функции $u(x, t) \in W_{2}^{0,1}\left(Q_{T}\right)$, производная которой $u_{t}(x, t) \in W_{2}^{1,0}\left(Q_{T}\right)$. Рассмотрим функцию $v(x, t) \in W_{2}^{1,0}\left(Q_{T}\right)$ вида

$$
v(x, t)=\sum_{k=1}^{M} a_{k}(t) \varphi_{k}(x) .
$$

Умножим уравнение $(5)$ на $a_{k}(t)$, просуммируем от 1 до $M$ и проинтегрируем от 0 до $T$. Получим

$$
\begin{gathered}
-\int_{Q_{T}} k(x) \nabla u_{t}^{N_{k}}(x, t) \nabla v(x, t) d x d t-\int_{Q_{T}} c(x) v(x, t) u_{t}^{N_{k}}(x, t) d x d t \\
=\int_{Q_{T}} b(x) v(x, t) u^{N_{k}}(x, t) d x d t+\int_{Q_{T}} f(x, t) v(x, t) d x d t .
\end{gathered}
$$

В силу приведенных вьше рассуждений можно перейти к пределу при $k \rightarrow \infty$. При этом получим

$$
\begin{gathered}
-\int_{Q_{T}} k(x) \nabla u_{t}(x, t) \nabla v(x, t) d x d t-\int_{Q_{T}} c(x) v(x, t) u_{t}(x, t) d x d t \\
=\int_{Q_{T}} b(x) v(x, t) u(x, t) d x d t+\int_{Q_{T}} f(x, t) v(x, t) d x d t
\end{gathered}
$$

То есть тождество из определения обобщенного решения верно для всех функций вида (8). Но эти линейные комбинации составляют в $W_{2}^{1,0}\left(Q_{T}\right)$ плотное множество. Следовательно, оно верно и при любой пробной функции $v(x, t) \in W_{2}^{1,0}\left(Q_{T}\right)$.

Согласно теоремам вложения из любой последовательности $\left\{u_{m}(x, t)\right\}$ такой, что нормы $\left\|u_{m}(x, t)\right\|_{W_{2}^{1}\left(Q_{T}\right)}$ ограничены, можно выбрать подпоследовательность, следы которой на $\Omega_{0}$ сходятся в $L_{2}\left(\Omega_{0}\right)$, сходящуюся в $L_{2}\left(\Omega_{0}\right)$. Значит, можно считать, что

$$
u^{N}(x, 0) \rightarrow u(x, 0) \quad \text { в } L_{2}\left(\Omega_{0}\right) .
$$

Причем $u(x, 0)=\varphi(x)$, так как $u^{N}(x, 0)=\sum_{k=1}^{\infty} c_{k}(0) \varphi_{k}(x) \rightarrow \varphi(x)$ в $L_{2}\left(\Omega_{0}\right)$ по свойствам ряда Фурье. Следовательно, $u(x, 0)=\varphi(x)$, т.е. функция $u(x, t)$, удовлетворяет определению обобщенного решения нашей задачи.

Доказательство единственности обобщенного решения проведем от противного. Предположим, что $u_{1}(x, t)$ и $u_{2}(x, t)$ - два различных решения нашей задачи. Тогда $u(x, t)=$ $u_{1}(x, t)-u_{2}(x, t)$ также является решением задачи $(1)-(3)$ с $f(x, t) \equiv 0$ в $Q_{T}$ и $\varphi(x) \equiv 0$ и удовлетворяет интегральному тождеству (5):

$$
\begin{aligned}
& -\int_{Q_{T}} k(x) \nabla u_{t}(x, t) \nabla v(x, t) d x d t-\int_{Q_{T}} c(x) v(x, t) u_{t}(x, t) x d t \\
& \quad=\int_{Q_{T}} b(x) v(x, t) u(x, t) d x d t+\int_{Q_{T}} f(x, t) v(x, t) d x d t \quad \forall v(x, t) \in W_{2}^{1,0}\left(Q_{T}\right) .
\end{aligned}
$$


Оценим $\|u(x, t)\|_{W_{2}^{0,1}\left(Q_{T}\right)}$ и $\left\|u_{t}(x, t)\right\|_{W_{2}^{1,0}\left(Q_{T}\right)}$. Для этого вместо $v(x, t)$ возьмем $u_{t}(x, t)$ и подставим в интегральное тождество. Подстановка корректна, так как по определению обобщенного решения $u_{t}(x, t) \in W_{2}^{1,0}\left(Q_{T}\right)$

$$
\begin{aligned}
& -\int_{Q_{T}} k(x)\left(\nabla u_{t}(x, t)\right)^{2} d x d t-\int_{Q_{T}} c(x)\left(u_{t}(x, t)\right)^{2} d x d t \\
& \quad=\int_{Q_{T}} b(x) u_{t}(x, t) u(x, t) d x d t .
\end{aligned}
$$

Отсюда легко получить следующие неравенства:

$$
\begin{aligned}
\left\|\nabla u_{t}(x, t)\right\|_{L_{2}\left(Q_{T}\right)}^{2} & \leqslant \frac{1}{2 k} B\|\varphi(x)\|_{L_{2}\left(\Omega_{0}\right)}^{2}+\frac{1}{2 c k}\|f(x, t)\|_{L_{2}\left(Q_{T}\right)}^{2}, \\
\left\|u_{t}(x, t)\right\|_{L_{2}\left(Q_{T}\right)}^{2} & \leqslant \frac{1}{c} B\|\varphi(x)\|_{L_{2}\left(\Omega_{0}\right)}^{2}+\frac{1}{c^{2}}\|f(x, t)\|_{L_{2}\left(Q_{T}\right)}^{2}, \\
\|u(x, t)\|_{L_{2}\left(Q_{T}\right)}^{2} & \leqslant \frac{B T}{b}\left(1+\frac{1}{c}\right)\|\varphi(x)\|_{L_{2}\left(\Omega_{0}\right)}^{2}+\frac{T}{b}\left(1+\frac{1}{c^{2}}\right)\|f(x, t)\|_{L_{2}\left(Q_{T}\right)}^{2} .
\end{aligned}
$$

Таким образом,

$$
\begin{aligned}
& \|u(x, t)\|_{W_{2}^{0,1}\left(Q_{T}\right)}^{2}=\|u(x, t)\|_{L_{2}\left(Q_{T}\right)}^{2}+\left\|u_{t}(x, t)\right\|_{L_{2}\left(Q_{T}\right)}^{2} \\
& \quad \leqslant B\left(\frac{T}{b}+\frac{T}{b c}+\frac{1}{c}\right)\|\varphi(x)\|_{L_{2}\left(\Omega_{0}\right)}^{2}+\left(\frac{1}{c^{2}}+\frac{T}{b}+\frac{T}{b c^{2}}\right)\|f(x, t)\|_{L_{2}\left(Q_{T}\right)}^{2}=0 .
\end{aligned}
$$

Следовательно, $u_{1}(x, t)=u_{2}(x, t)$, т.е. решение задачи единственно.

\section{СПИСОК ЦИТИРОВАННОЙ ЛИТЕРАТУРЫ}

[1] Свиридюк Г. А. К общей теории полугрупп // УМН. 1994. Т. 49. №4. С. 47-74.

[2] Свиридюк Г. А., Сукачева Т. Г. Задача Коши для одного класса полулинейных уравнений типа Соболева // Сиб. матем. ж. 1990. Т. 31. № 5. С. 109-119.

[3] Ушаков В. И. Стабилизация решений третьей смешанной задачи для параболического уравнения второго порядка в нецилиндрической области // Матем. сб. 1980. Т. 3. № 1. С. 95-115.

[4] Иванова М. В. Вторая краевая задача для уравнения типа Соболева в нецилиндрической области // "Понтрягинские чтения-Х". Тез. докл. Воронеж: ВГУ, 1999. С. 114.

Челябинский государственный университет 